\title{
Differences in Pertussis Incidence by Income among Oregon Teens during an Outbreak
}

\author{
Steve G. Robison, Juventila Liko, and Paul R. Cieslak \\ Oregon Immunization Program, Oregon Health Authority, 800 NE Oregon Street, Portland, OR 97232, USA \\ Correspondence should be addressed to Steve G. Robison; steve.g.robison@state.or.us
}

Received 29 August 2015; Revised 13 November 2015; Accepted 16 November 2015

Academic Editor: Wagner Quintilio

Copyright ( 2015 Steve G. Robison et al. This is an open access article distributed under the Creative Commons Attribution License, which permits unrestricted use, distribution, and reproduction in any medium, provided the original work is properly cited.

That disease and poverty are connected is a cornerstone of public health thought. In the case of pertussis, however, it is possible that the expected relationship to poverty is reversed. Grounds exist for considering that increases in income are associated with increases in pertussis rates, both in terms of real risk through social and network features and through the possibility of greater likelihood of care seeking and detection based on income. Using reported adolescent pertussis cases from a 2012 outbreak in Oregon, pertussis incidence rates were determined for areas grouped by zip code into higher, middle, and lower median household income. Adolescents of ages 13-16 years in higher income areas were 2.6 times (95\% CI 1.8-3.8) more likely as all others to have reported pertussis during the 2012 outbreak and 3.1 (95\% CI 1.4-6.5) times as likely as those in lower income areas. The higher pertussis rates associated with higher income areas were observed regardless of Tdap rate differences. These results suggest that income may be associated with disease risk, likelihood of diagnosis and reporting, or both. Further evaluation of this finding is warranted.

\section{Introduction}

A feature of recent pertussis outbreaks in the United States has been an increased number of cases reported among teenagers. According to the U.S. Centers for Disease Control and Prevention (CDC), 29.9\% of cases in 2012 occurred in those of 11-19 years of age [1]. The near elimination of childhood pertussis has been one of the success stories of vaccination, with annual disease incidence dropping from 157 cases per 100,000 in the prevaccine era to $<1$ per 100,000 in the 1970s [2]. Another worrisome feature of recent outbreaks is that many teens have become ill despite Tdap immunization. Among the hypotheses suggested to explain the resurgence in teen pertussis are rapid waning of immunity from pertussis vaccination, inadequate herd immunity due to vaccine refusal, increased testing and reporting of pertussis, shifts from whole cell to acellular vaccine, and genetic drift of pertussis strains that skirt vaccine-derived immunity [3-8].

It is also possible that sociodemographic features such as income may affect reported rates of pertussis. It is likely that pertussis among both adolescents and adults in the US is endemic but largely underreported $[9,10]$. Higher income may influence who is more likely to seek care or to receive testing for nonspecific respiratory diseases, including many cases of pertussis. But it is also possible that pertussis incidence may really be higher among higher income cohorts because of differential vaccination rates and associated immunity, or contact structures and social networks that make exposure more likely.

The purpose of this analysis was to examine whether local area differences in income levels were associated with differences in reported adolescent pertussis disease rates in Northwest Oregon during a 2012 outbreak. A secondary concern was whether income levels affected apparent disease risk independent of community Tdap immunization rates for adolescents.

\section{Methods}

Immunization and demographic records from the Oregon ALERT Immunization Information System (ALERT IIS) were linked to reported pertussis case data for 2012 in Oregon. ALERT IIS is populated by Oregon birth records and by 
TABle 1: 2012 Northwest Oregon teen pertussis by income categories.

\begin{tabular}{|c|c|c|c|}
\hline & \multicolumn{3}{|c|}{ Zip code income stratum } \\
\hline & Lower & Middle & Upper \\
\hline Zip code $N$ & 22 & 95 & 21 \\
\hline Population (age 13-16) & 12,416 & 72,108 & 23,905 \\
\hline Median household income & 35,827 & 54,303 & 77,940 \\
\hline Tdap immunization rate (age 13-16) & $85.40 \%$ & $88 \%$ & $88.10 \%$ \\
\hline VFC pertussis immunization & $33.70 \%$ & $25.80 \%$ & $12.50 \%$ \\
\hline Median time since Tdap (years) & 2.87 & 2.97 & 3.12 \\
\hline Pertussis cases (age 13-16) & 8 & 57 & 47 \\
\hline Cases $/ 1,000$ & 0.64 & 0.79 & 1.97 \\
\hline Risk ratio \& 95\% CI & 1.0 (ref.) & $1.23(0.59-2.57)$ & $3.05(1.44-6.46)$ \\
\hline
\end{tabular}

immunization and demographic records received from $>95 \%$ of Oregon childhood vaccine providers. Because the 2012 Oregon pertussis outbreak occurred primarily in the northwest part of the State, the study population was restricted to those with last known addresses in a contiguous, ninecounty region centered in metropolitan Portland: Clackamas, Clatsop, Columbia, Marion, Multnomah, Polk, Tillamook, Washington, and Yamhill counties. Data for both pertussis cases and noncases were compiled for this region by zip code, with immunization status ascertained both from case investigation and from ALERT IIS. Cases were classified as "confirmed" according to the 2010 definition established by the Council of State and Territorial Epidemiologists [11].

Data on income distribution by zip code were downloaded from the Population Estimates Program of the U.S. Census Bureau. Lower income zip codes were defined as those where the percentage of households with annual incomes of $<\$ 35,000$ exceeded the mean by at least one standard deviation. Higher income zip codes were defined as those where the percentage of households with incomes $>\$ 100,000 /$ year exceeded the mean by at least one standard deviation. Middle income zip codes were defined as those meeting neither of the above criteria. While these criteria are not necessarily mutually exclusive, in our study each zip code fell into only one income category. Zip code areas with $<10$ adolescents or for which there was no match between Census and postal zip code lists were excluded from the analysis; this exclusion did not remove any pertussis cases from the study. Population counts per zip code of adolescents aged 13 to 16 were based on adjusted ALERT IIS reporting [12].

Pertussis incidence rates were calculated for each of the three zip code income groups. Risk ratios and confidence intervals were calculated in WinPepi [13] for the middle and more affluent income zip codes, with incidence in the lower income zip codes as the referent. Using ALERT IIS data, Tdap immunization rates among teens $13-16$ years of age were also calculated by zip code and compared to disease rates. In addition, the percentage of adolescents with any record of receipt of pertussis-containing vaccine through the Vaccines for Children Program (VFC) was examined by zip code as an alternate proxy for income. Higher income areas based on VFC were defined as where less than $1 / 6$ th of teens had used any VFC pertussis vaccine, and low income areas were defined as where more than $1 / 3$ rd had used VFC pertussis vaccine. The VFC program in the US provides free vaccine to low income and disadvantaged children. To complement the area-based analysis, an individual-level analysis of pertussis risk was conducted for the cohort of 13-year-olds in 2012, stratifying by participation in the Oregon Health Plan (OHP), which is limited to those with family incomes $<200 \%$ of the U.S. Federal Poverty Level. OHP participation was used as a proxy for lower family income.

\section{Results}

Based on ALERT IIS records, a total of 109,080 teenagers 13-16 years of age resided in the 138 zip code areas in the nine study counties of Northwest Oregon. Among the 138 zip code areas used in the analysis, $16 \%$ were categorized as lower income, $15 \%$ as higher income, and $69 \%$ as middle income. Population and pertussis data for the zip code income areas are presented in Table 1. During 2012, 112 confirmed pertussis cases from 43 of the 138 zip codes were reported among teens 13-16 years of age. The mean Tdap immunization rate was $85.4 \%$ in lower income areas, $88.0 \%$ in middle income areas, and $88.1 \%$ in higher income areas.

Residence in a zip code with a Tdap immunization rate above the median ( $R R=0.8,95 \%$ CI 0.6-1.2) was not significantly associated with lower reported pertussis incidence. Overall, adolescents living in higher income zip codes were 2.6 (95\% CI 1.8-3.8) times more likely to have reported pertussis in 2012 as those in other zip codes and 3.1 (95\% CI 1.4-6.5) times more likely as those in the lower income category. The association of pertussis incidence with residence in higher income zip codes persisted after zip code stratification by Tdap immunization rate. Among zip codes with teen Tdap immunization rates above the median, those in higher income zip codes were 2.5 (95\% CI 1.2-5.5) times more likely than all others to have reported pertussis. Among those resident in zip code areas with Tdap rates below the median, those in higher income areas were also 2.7 (95\% CI 1.7-4.4) times more likely than all others to have reported pertussis.

For the alternative zip code area categorization by VFC percentages, a similar relationship to pertussis was observed as that found from income grouping. Zip code areas with low VFC usage had pertussis incidence rates that were 2.1 
(95\% CI 1.3-4.0) higher than rates in high VFC usage areas. VFC usage was also observed to vary by area incomes. In higher income zip code areas, the mean rate of adolescents using VFC vaccine for Tdap was $12.5 \%$; in middle income areas, this rate increased to $25.8 \%$, and in lower income areas it was $33.7 \%$.

As a confirmatory analysis of individual risk by household income, 26,760 teenagers who turned 13 years of age during 2012 were evaluated for pertussis risk against income. Overall, $25.5 \%$ of this population was enrolled in the Oregon Health Plan (OHP), for whom in 2012 eligibility is restricted to those with family incomes under $200 \%$ of the US poverty level. Of 46 pertussis cases among Northwest Oregon adolescents 13 years of age, $5(11 \%)$ occurred among OHP clients. The incidence rate among OHP 13-year-olds was 0.74/1,000, compared to 2.06/1,000 among non-OHP 13-year-olds $(\mathrm{RR}=$ $0.4,95 \%$ CI $0.1-0.9)$.

\section{Discussion}

This study found that more affluent areas experienced higher reported pertussis rates among adolescents during a 2012 outbreak. This increased reported risk occurred irrespective of the differences in area immunization levels. On an individual level, this study also found that lower income status as measured by participation in the Oregon Health Plan was associated with lower reported pertussis rates. Other studies have shown, using reported case data, that Tdap immunization is protective against pertussis [14, 15]. Our findings in no way conflict with the strategy of building pertussis herd immunity with Tdap, but they do present a caveat to the interpretation of vaccine effectiveness and pertussis reports across heterogeneous populations.

The risk of pertussis disease for an individual is a combination of exposure and vulnerability. The chances of capturing a case of pertussis are based on the likelihood of care seeking and testing. From the data shown here, it is not known whether the increase in reporting risk that was observed based on income is from a real difference in disease due to increased exposure or from greater identification of disease due to increased chance of seeking care and testing. Basic immunization rates and well child visit rates in Oregon are similar for higher and lower income populations, but whether care seeking and testing for respiratory illness vary by income is unknown.

Differences in care seeking and testing could account for observed differences in rates of reported pertussis by income. Alternatively, variability of exposure, in terms of proximity, duration, or respiratory hygiene, or differential susceptibility to pertussis following exposure based on socioeconomic factors rather than on vaccine-induced immunity, may be playing a role. Income and social class have been shown to affect numbers and types of interpersonal contacts, leading to a potential for a real difference in disease risk based on income across social networks [16]. Another possibility is that social networks among those of relative affluence include more children who are missing immunizations. Pertussis incidence in younger children, based on parental choices to limit or avoid immunizations, is associated with higher parental socioeconomic status [17-19]. A few studies to date have shown that those in a social network are more likely to have similar vaccination status and that disease can spread predominantly through more vulnerable social networks with lesser, if any, effects on more protected social networks [20-22].

Geographic or social clusters of parents who decline to immunize their children in a timely manner can create pockets of vulnerability to disease. One study of the 2010 pertussis outbreak in California found an association between clusters of parental exemptions to school-required immunizations and areas with higher pertussis rates [23]. Other studies have shown an association between measles risk and parental choice not to vaccinate, finding correlations among higher income, nonvaccination, and disease in localized outbreaks [18].

Mobility that occurred between when Tdap is due at ages 11 to 13 and when coverage rates were assessed at ages 11 to 17 may introduce a small amount of errors into calculated vaccine coverage. Zip code coverage rates used in this study were based on adolescents' last known addresses in the ALERT IIS, where addresses are typically updated with every immunization visit. Whether this last known address is the actual current residence is unknown, and between 3 and 5\% of the study population is expected to move in any year. Although the authors are not aware of any patterns of mobility in the study area that could bias coverage rates, this is a topic in need of further research.

A number of potential limitations may apply to this study. Different methods of stratifying the study area for income could have been employed. Zip code areas are a crude proxy for community, and teens are likely to cross zip code boundaries in congregating for school, shopping, and social events. Finally, pertussis rates in a single age band for adolescents may be influenced by the presence of disease in persons of both younger and older ages, a topic outside of the scope of this study.

\section{Conclusion}

During a 2012 outbreak in Oregon, residence in higher income zip codes was associated with increased levels of reported pertussis among adolescents of 13-16 years of age. The relationship between surrogates of household income and reported pertussis was observed on both aggregated geographic basis and individual teenagers stratified by Oregon Health Plan participation. Whether the connection between pertussis and income is due to differences in care seeking and diagnostic testing, or to real disease risk differences, is unknowable from the present information. Further research is warranted to the causes of the observed income effect.

\section{Disclaimer}

The paper's contents are solely the responsibility of the authors and do not necessarily represent the official views of the Centers for Disease Control and Prevention or the Department of Health and Human Services. 


\section{Conflict of Interests}

The authors declare that there is no conflict of interests regarding the publication of this paper.

\section{Acknowledgments}

The authors of this study wish to acknowledge the contributions of Mary Beth Kurilo, for assistance in design and oversight; Kathy Scott, for review of drafts; and Andrew Osborn, for data assistance. This paper was supported by Grants 1H23IP000767-01 and 5H23Ip000785-02, and Cooperative Agreement no. CK000313-03 (Emerging Infections Program) funded by the Centers for Disease Control and Prevention.

\section{References}

[1] U.S. Centers for Disease Control (CDC), "2012 final pertussis surveillance report," http://www.cdc.gov/pertussis/downloads/ pertussis-surveillance-report.pdf.

[2] J. D. Cherry, "The present and future control of pertussis," Clinical Infectious Diseases, vol. 51, no. 6, pp. 663-667, 2010.

[3] J. D. Cherry, "Pertussis: challenges today and for the future," PLoS Pathogens, vol. 9, no. 7, Article ID e1003418, 2013.

[4] N. P. Klein, J. Bartlett, A. Rowhani-Rahbar, B. Fireman, and R. Baxter, "Waning protection after fifth dose of acellular pertussis vaccine in children," The New England Journal of Medicine, vol. 367, no. 11, pp. 1012-1019, 2012.

[5] A. M. Queenan, P. K. Cassiday, and A. Evangelista, "Pertactinnegative variants of Bordetella pertussis in the United States," The New England Journal of Medicine, vol. 368, no. 6, pp. 583-584, 2013.

[6] J. Liko, S. G. Robison, and P. R. Cieslak, "Priming with wholecell versus acellular pertussis vaccine," The New England Journal of Medicine, vol. 368, no. 6, pp. 581-582, 2013.

[7] S. L. Sheridan, R. S. Ware, K. Grimwood, and S. B. Lambert, "Number and order of whole cell pertussis vaccines in infancy and disease protection," The Journal of the American Medical Association, vol. 308, no. 5, pp. 454-456, 2012.

[8] A. M. Acosta, C. DeBolt, A. Tasslimi et al., "Tdap vaccine effectiveness in adolescents during the 2012 Washington State pertussis epidemic," Pediatrics, vol. 135, no. 6, pp. 981-989, 2015.

[9] A. A. McGirr, A. R. Tuite, and D. N. Fisman, "Estimation of the underlying burden of pertussis in adolescents and adults in southern Ontario, Canada," PLoS ONE, vol. 8, no. 12, Article ID e83850, 2013.

[10] J. D. Cherry, "The history of pertussis (whooping cough); 19062015: facts, myths, and misconceptions," Current Epidemiology Reports, vol. 2, no. 2, pp. 120-130, 2015.

[11] U.S. Centers for Disease Control (CDC), "Pertussis (Whooping Cough) (Bordetella pertussis): 2010 case definition," http://wwwn .cdc.gov/NNDSS/script/casedef.aspx?CondYrID=795\&Date$\mathrm{Pub}=1 / 1 / 2010 \% 2012: 00: 00 \% 20 \mathrm{AM}$.

[12] S. G. Robison, "Addressing immunization registry population inflation in adolescent immunization rates," Public Health Reports, vol. 130, no. 2, pp. 161-167, 2015.

[13] J. H. Abramson, "WINPEPI updated: computer programs for epidemiologists, and their teaching potential," Epidemiologic Perspectives \& Innovations, vol. 8, no. 1, article 1, 2011.
[14] R. S. Barlow, L. E. Reynolds, P. R. Cieslak, and A. D. Sullivan, "Vaccinated children and adolescents with pertussis infections experience reduced illness severity and duration, Oregon, 2010 - 2012," Clinical Infectious Diseases, vol. 58, no. 11, pp. 15231529, 2014.

[15] J. Liko, S. G. Robison, and P. R. Cieslak, "Pertussis vaccine performance in an epidemic year-Oregon, 2012," Clinical Infectious Diseases, vol. 59, no. 2, Article ID ciu273, pp. 261-263, 2014.

[16] M. Savage, F. Devine, N. Cunningham et al., "A new model of social class? Findings from the BBC's Great British Class Survey experiment," Sociology, vol. 47, no. 2, pp. 219-250, 2013.

[17] S. B. Omer, K. S. Enger, L. H. Moulton, N. A. Halsey, S. Stokley, and D. A. Salmon, "Geographic clustering of nonmedical exemptions to school immunization requirements and associations with geographic clustering of pertussis," American Journal of Epidemiology, vol. 168, no. 12, pp. 1389-1396, 2008.

[18] D. E. Sugerman, A. E. Barskey, M. G. Delea et al., "Measles outbreak in a highly vaccinated population, San Diego, 2008: role of the intentionally undervaccinated," Pediatrics, vol. 125, no. 4, pp. 747-755, 2010.

[19] D. R. Feikin, D. C. Lezotte, R. F. Hamman, D. A. Salmon, R. T. Chen, and R. E. Hoffman, "Individual and community risks of measles and pertussis associated with personal exemptions to immunization," Journal of the American Medical Association, vol. 284, no. 24, pp. 3145-3150, 2000.

[20] P. Rohani, X. Zhong, and A. A. King, "Contact network structure explains the changing epidemiology of pertussis," Science, vol. 330, no. 6006, pp. 982-985, 2010.

[21] M. Dickison, S. Havlin, and H. E. Stanley, "Epidemics on interconnected networks," Physical Review E, vol. 85, no. 6, Article ID 066109, 2012.

[22] V. C. Barclay, T. Smieszek, J. He et al., "Positive network assortativity of influenza vaccination at a high school: implications for outbreak risk and herd immunity," PLOS ONE, vol. 9, no. 2, Article ID e87042, 2014.

[23] J. E. Atwell, J. Van Otterloo, J. Zipprich et al., "Nonmedical vaccine exemptions and pertussis in California, 2010," Pediatrics, vol. 132, no. 4, pp. 624-630, 2013. 

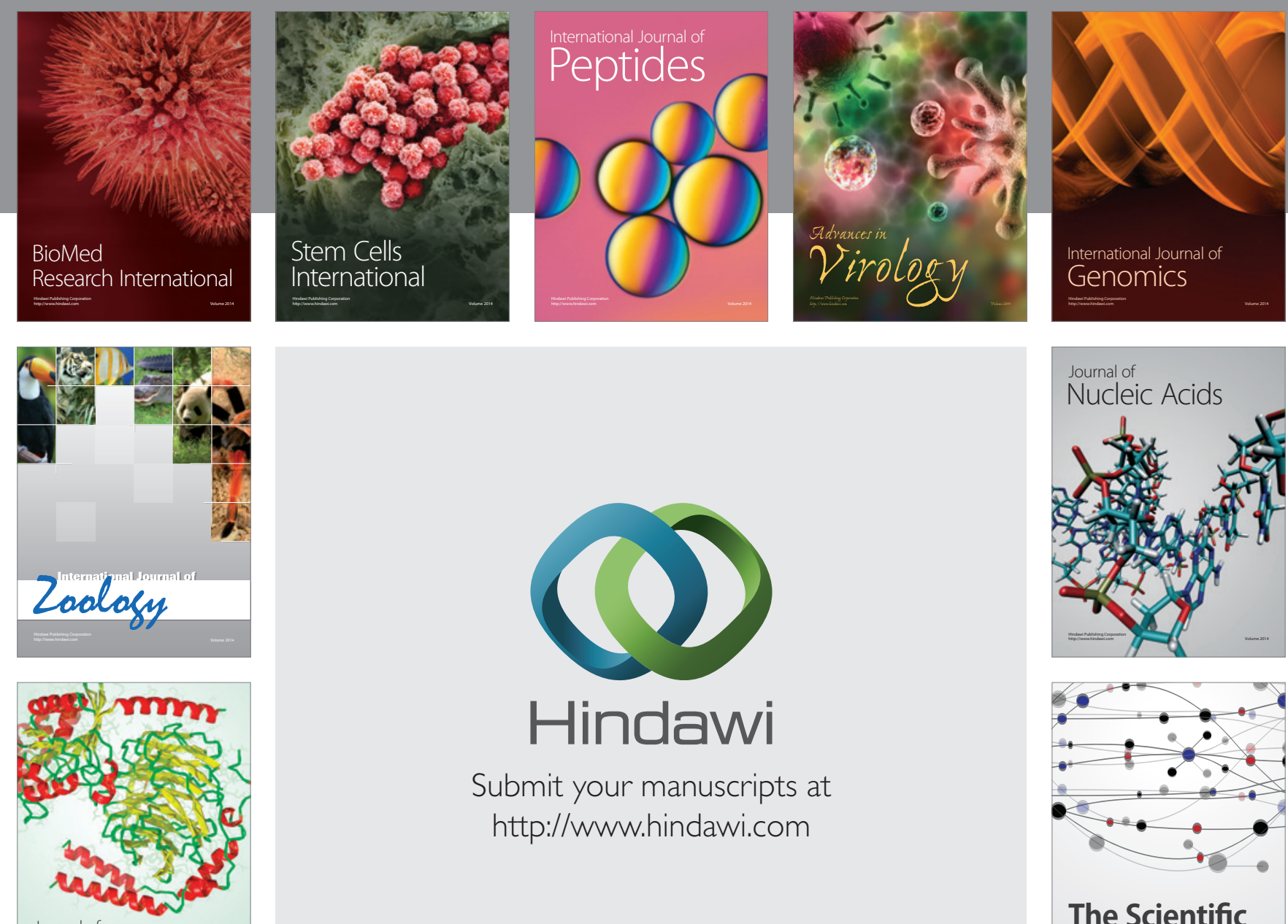

Submit your manuscripts at

http://www.hindawi.com

Journal of
Signal Transduction
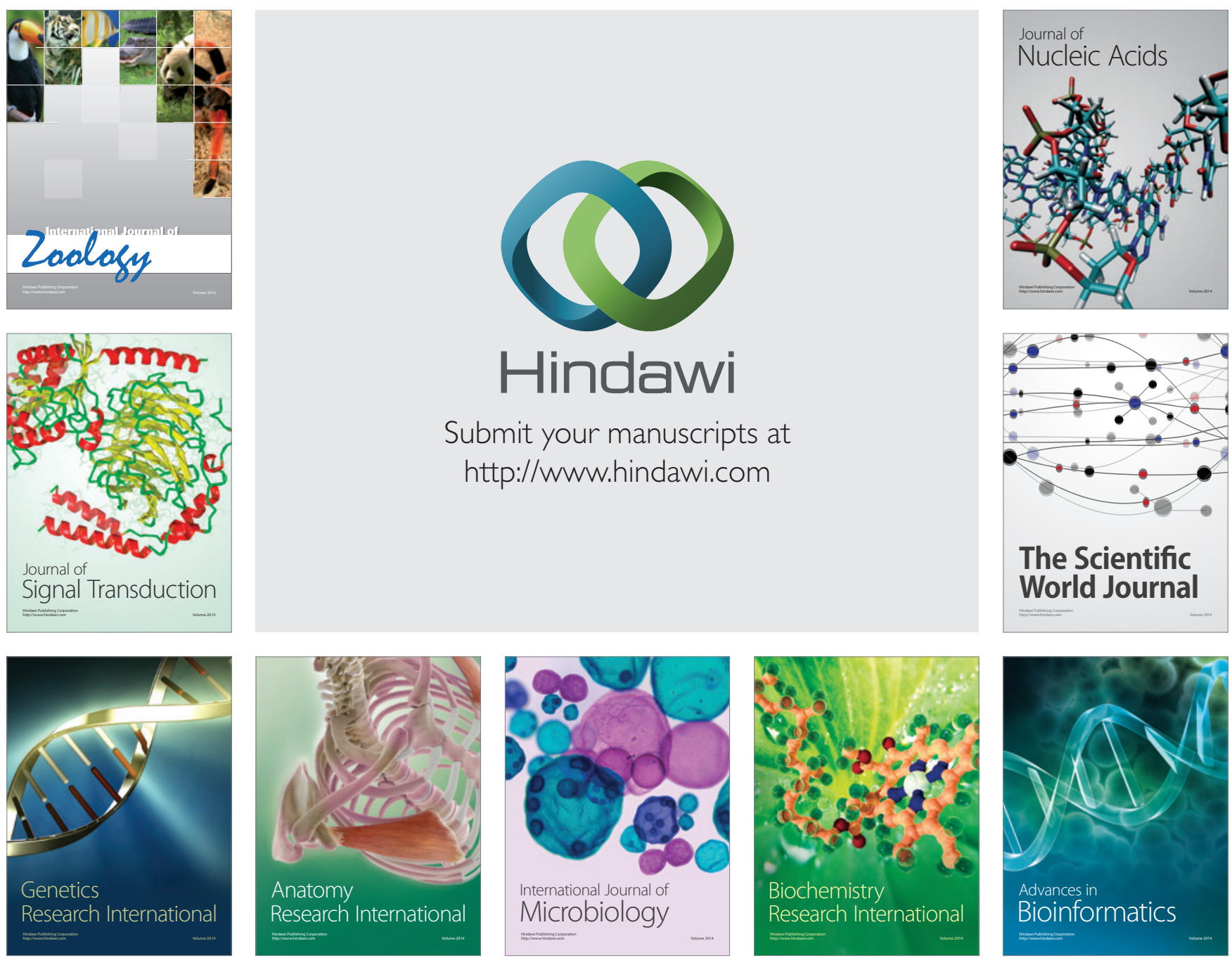

The Scientific World Journal
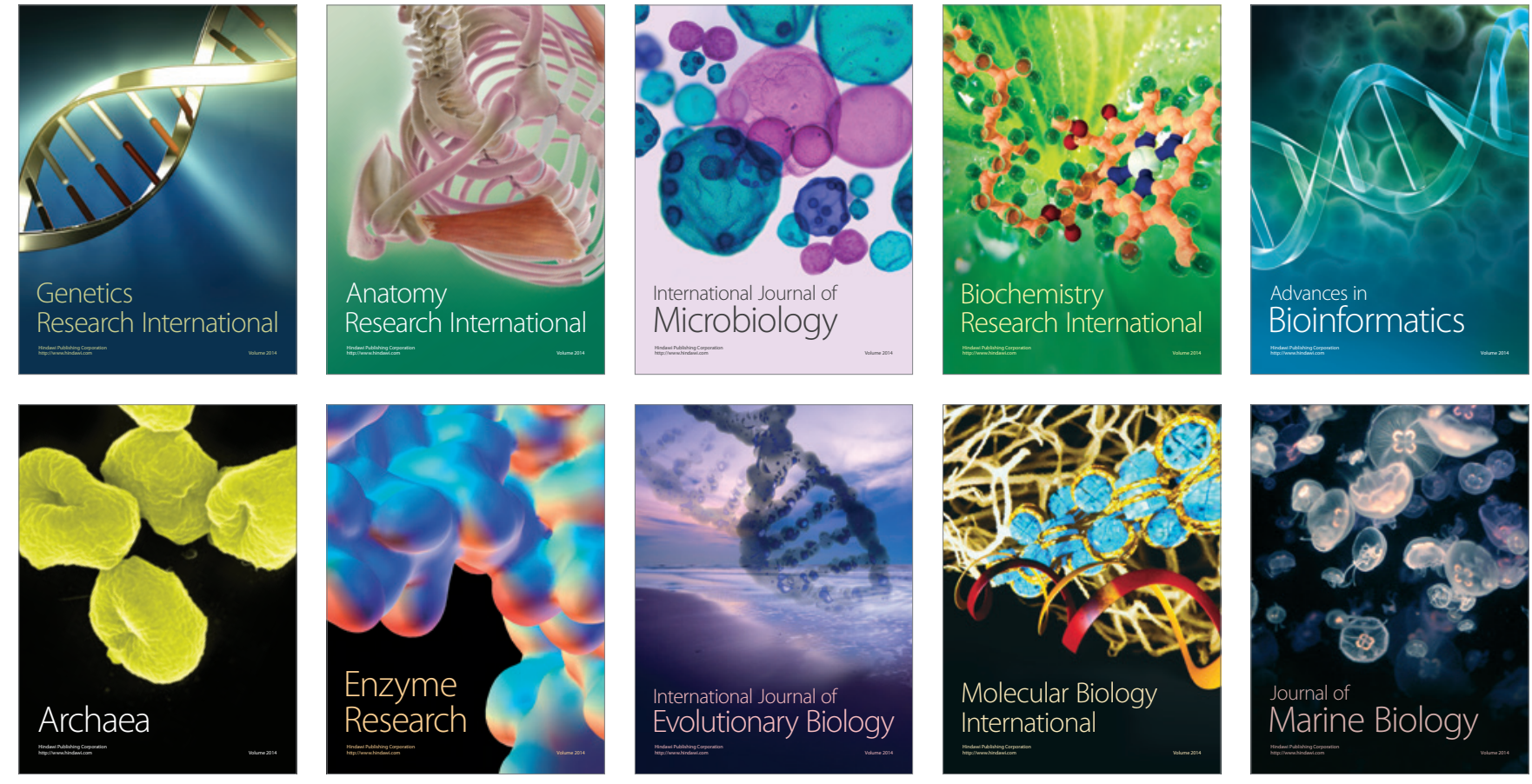